Proyecciones Journal of Mathematics

Vol. 30, No 1, pp. 19-28, May 2011.

Universidad Católica del Norte

Antofagasta - Chile

\title{
Bipartite theory of irredundant set
}

\author{
V. SWAMINATHAN \\ S. N. COLLEGE, INDIA \\ and \\ Y. B. VENKATAKRISHNAN \\ SASTRA UNIVERSITY, INDIA \\ Received : June 2010. Accepted : December 2010
}

\begin{abstract}
The bipartite version of irredundant set, edge-vertex irredundant set and vertex-edge irredundant set are introduced. Using the bipartite theory of graph, $I R_{v e}(G)+\gamma(G) \leq|V|$ and $\gamma_{v e}(G)+I R(G) \leq|V|$ are proved.
\end{abstract}

AMS classification : $05 C 69$

Keywords : Bipartite graph, $X$-irredundant set, Hyper $Y$-irredundant set, edge-vertex and vertex-edge irredundant sets. 


\section{Introduction}

All graphs considered here are simple and undirected. [4,5] suggests that given any problem, say $\mathrm{P}$, on an arbitrary graph $G$, there is very likely a corresponding problem $\mathrm{Q}$ on a bipartite graph $G^{1}$, such that a solution for $\mathrm{Q}$ provides a solution for P. The bipartite theory of graphs was introduced in [4] and a parameter called $X$-domination number of a bipartite graph was defined. Let $G=(X, Y, E)$ be a bipartite graph with $|X|=p$ and $|Y|=q$. Two vertices $u$ and $v$ in $X$ are $X$-adjacent if they have a common adjacent vertex $y \in Y$. Let $y \in X$ and $\Delta_{Y}=\max \left\{\left|N_{Y}(u)\right|: y \in X\right\}$ where the $X$-neighbor set $N_{Y}(u)$ is defined as $N_{Y}(u)=\{v \in X: u$ and $v$ are $X-$ adjacent\}.

A subset $X \subseteq X$ is an $X$-dominating set [4] if every $x \in X-D$ is $X$-adjacent to some vertex in $D$. The minimum cardinality of a $X$-dominating set is called $X$-domination number and is denoted by $\gamma_{X}(G)$.

We say a vertex $x \in X$ hyper $Y$-dominates $y \in Y$ if $y \in N(x)$ or $y \in N\left(N_{Y}(x)\right)$. A subset $S \subseteq X$ is a hyper $Y$-dominating set [6] if every $y \in Y$ is hyper $Y$-dominated by a vertex of $S$. The minimum cardinality of a hyper $\mathrm{Y}$-dominating set is called hyper $Y$-domination number and is denoted by $\gamma_{h Y}(G)$.

Given an arbitrary graph $G=(V, E)$, a vertex $u \in V(G)$ ve-dominates an edge $v w \in E(G)$ if (a) $u=v$ or $u=w$ ( $u$ incident to $v w$ ) or (b) $u v$ or $u w$ is an edge in $G$. A subset $S \subseteq V(G)$ is a vertex-edge dominating set [3] if for all edges $e \in E(G)$, there exists a vertex $v \in S$ such that $v$ dominates $e$. The minimum cardinality of a ve-dominating set of $G$ is called the vertex-edge domination number and is denoted as $\gamma_{v e}(G)$.

An edge $e=u v \in E(G)$ ev-dominates a vertex $w \in V(G)$ if (i) $u=w$ or $v=w(w$ is incident to $e$ ) or (ii) $u w$ or $v w$ is an edge in $G$. ( $w$ is adjacent to $u$ or $v)$. A set $S \subseteq E(G)$ is an edge-vertex dominating set [3] if for all vertices $v \in V(G)$, there exists an edge $e \in S$ such that $e$ dominates $v$. The minimum cardinality of a ev-dominating set of $G$ is called the edge-vertex domination number and is denoted as $\gamma_{e v}(G)$.

Observation: 1. Let $G$ be an arbitrary graph. A vertex $u \in V(G)$ vedominates the edge $e \in E(G)$ if and only if the edge $e$ ev-dominates the vertex $u \in V(G)$. 


\section{Bipartite Construction}

The bipartite graph $V E(G)$ constructed from an arbitrary graph $G=$ $(V, E)$ is defined as in [4]. $V E(G)=(V, E, F)$ is defined by the edges $F=\{(u, e): e=(u, v) \in E\} \cdot V E(G) \cong S(G)$, where $S(G)$ denotes the subdivision graph of $G$.

The bipartite graph $E V(G)[4]$ constructed from an arbitrary graph $G=(V, E)$ is defined as $E V(G)=(E, V, J)$ where $J=\{(e, u)(e, v): e=$ $(u, v) \in E\}$.

A set $S \subseteq V$ of vertices in a graph $G=(V, E)$ is called a dominating set [2] if every $v \in V$ is either an element of $S$ or is adjacent to an element of $S$. The minimum cardinality of a dominating set of a graph $G$ is called the domination number and is denoted by $\gamma(G)$.

A set $F \subseteq E(G)$ of edges in a graph $G=(V, E)$ is called an edge dominating set [2] if every $e \in E(G)$ is either an element of $F$ or is adjacent to an element of $E-F$. The minimum cardinality of an edge dominating set of a graph $G$ is called the edge domination number and is denoted by $\gamma_{1}(G)$.

Theorem:2.1 [4] For any graph $G$,

(a) $\gamma_{X}(V E(G))=\gamma(G)$

(b) $\gamma_{X}(E V(G))=\gamma_{1}(G)$.

Theorem:2.2 [6] For any graph $G$,

(a) $\gamma_{h Y}(V E(G))=\gamma_{v e}(G)$

(b) $\gamma_{h Y}(E V(G))=\gamma_{e v}(G)$.

\section{Irredundant sets}

\subsection{Vertex-edge irredundant set}

A vertex $v \in S \subseteq V(G)$ has a private edge $e=u w \in E(G)$ (with respect to a set $S$ ), if: 1 . $v$ is incident to $e$ or $v$ is adjacent to either $u$ or $w$, and 2 . for every vertices $x \in S-\{v\}, x$ is not incident to $e$ and $x$ is not adjacent to either $u$ or $w$.

A set $S$ is a vertex-edge irredundant set [3] (simply a ve-irredundant set) if every vertex $v \in S$ has a private edge. The vertex-edge irredundance of a graph $G$ is the cardinality of a maximal ve-irredundant set with minimum number of vertices and is denoted by $i r_{v e}(G)$. The upper vertexedge irredundance number of a graph $G$ is the cardinality of a maximum 
ve-irredundant set of vertices and is denoted by $I R_{v e}(G)$.

Theorem: 3.1.1 [3] Every minimal ve-dominating set is a maximal veirredundant set.

\subsection{Edge-vertex irredundant set}

An edge $e=u v \in F \subseteq E(G)$ has a private vertex $w \in V(G)$ ( with respect to a set $F$ ), if: 1 . $e$ is incident to $w$, and 2. for all edges $f=x y \in F-\{e\}$, $f$ is not incident to $w$ and neither $x$ nor $y$ is adjacent to $w$.

A set $F$ is an edge-vertex irredundant set [3] ( simply a ev-irredundant set) if every edge $e \in F$ has a private vertex. The edge-vertex irredundance of a graph $G$ is the cardinality of a maximal ev-irredundant set with minimum number of vertices and is denoted by $i r_{e v}(G)$. The upper edgevertex irredundance number of a graph $G$ is the cardinality of a maximum ev-irredundant set of vertices and is denoted by $I R_{e v}(G)$.

Theorem 3.2.1:[3] Every minimal ev-dominating set of $G$ is a maximal ev-irredundant set.

\subsection{Hyper $Y$ - Irredundant set}

Let $G=(X, Y, E)$ be a bipartite graph. Let $S \subseteq X$. A vertex $x \in S$ has a private hyper $Y$-neighbor $y \in Y$ if 1 . $x$ is adjacent to $y$ or $y \in$ $N\left(N_{Y}(x)\right)$ and 2. for all vertices $x_{1} \in S-\{x\}, x_{1}$ is not adjacent to $y$ and $y \notin N\left(N_{Y}\left(x_{1}\right)\right)$.

A set $S$ is hyper $Y$-irredundant set if every $v \in S$ has a private hyper $Y$-neighbor. The hyper $Y$-irredundance number of a graph $G$ is the minimum cardinality of a maximal hyper $Y$-irredundant set of vertices and is denoted by $\operatorname{ir}_{h Y}(G)$. The upper hyper $Y$-irredundance number of a graph $G$ is the maximum cardinality of a maximal hyper $Y$-irredundant set of vertices and is denoted by $I R_{h Y}(G)$.

Theorem: 3.3.1 A hyper $Y$-dominating set $S$ is a minimal hyper $Y$-dominating set if and only if it is hyper $Y$-dominating set and hyper $Y$-irredundant set. 
Proof: Let $S$ be a hyper $Y$-dominating set. Then $S$ is a minimal hyper $Y$-dominating set if and only if $\forall u \in S, \exists y \in Y$ which is not hyper $Y$ dominated by $S-\{u\}$. Equivalently, $S$ is a minimal hyper $Y$-dominating set if and only if $\forall u \in S, u$ has atleast one private hyper $Y$-neighbour. Thus $\mathrm{S}$ is minimal hyper $Y$-dominating set if and only if it is hyper $Y$-irredundant set.

Conversely, let $S$ be both hyper $Y$-dominating and hyper $Y$-irredundant.

Claim: $S$ is a minimal hyper $Y$-dominating set.

If $S$ is not minimal hyper $Y$-dominating set, there exists $v \in S$ for which $S-\{v\}$ is hyper $Y$-dominating. Since $S$ is hyper $Y$-irredundant, $v$ has a private hyper $Y$-neighbor of $u$. By definition $u$ is not hyper $Y$-adjacent to any vertex in $S-\{v\}$. That is, $S-\{v\}$ is not hyper $Y$-dominating set, a contradiction. Hence, $S$ is a minimal hyper $Y$-dominating set.

Theorem: 3.3.2 Every minimal hyper $Y$-dominating set is a maximal hyper $Y$-irredundant set.

Proof: Every minimal hyper $Y$-dominating set $S$ is hyper $Y$-irredundant set.

Claim: $S$ is a maximal hyper $Y$-irredundant set.

Suppose $S$ is not maximal hyper $Y$-irredundant set. Then there exists a vertex $u \in X-S$ for which $S \cup\{u\}$ is hyper $Y$-irredundant. There exists atleast one vertex $y \in Y$ which is a private hyper $Y$-neighbor of $u$ with respect to $S \cup\{u\}$. That is no vertex in $S$ is hyper $Y$-adjacent to $y$. Hence, $S$ is not a hyper $Y$-dominating set, a contradiction. Hence, $S$ is a maximal hyper $Y$-irredundant set.

Theorem:3.3.3 For any graph $G$,

(a) $i r_{h Y}(V E(G))=i r_{v e}(G)$

(b) $i r_{h Y}(E V(G))=i r_{e v}(G)$.

Proof: Let $S$ be a $i r_{h Y}$-set of $V E(G)=(X, Y, E)$. Every $x \in S$ has a private hyper $Y$-neighbor $y \in Y . x$ is adjacent to $y$ or $y \in N\left(N_{Y}(x)\right)$ and for all vertices $x_{1} \in S-\{x\}, x_{1}$ is not adjacent to $y$ and $y \notin N\left(N_{Y}\left(x_{1}\right)\right)$. In graph $G, x \in S \subseteq V$ is incident with $y \in E$ or $x$ is adjacent to either $u$ or $v$ where $y=u v$ and for every $x_{1} \in S-\{x\}, y \in E$ is not incident with 
$x_{1}$ and $x_{1}$ is not adjacent to either $u$ or $v . S$ is a vertex edge irredundant set.

$i r_{e v}(G) \leq|S|=i r_{h Y}(V E(G))$.

Let $U$ be a $i r_{v e}$-set of $G$. Every vertex $v \in S$ has a private edge $e=u w$ with respect to $U$. Equivalently, $v$ is incident with $e$ or $v$ is adjacent to either $u$ or $w$ and for every $x \in U-\{v\}, x$ is not incident with $e$ and $x$ is not adjacent to either $u$ or $w$. In $V E(G)$, every $v \in S$ has private hyper $Y$-neighbor $e$. Therefore, $U \subseteq X$ is a hyper $Y$-irredundant set of $V E(G)$. Hence, $i r_{h Y}(V E(G)) \leq|U|=i r_{v e}(G)$.

Similarly (b) can be proved.

\subsection{X-Irredundant set}

Let $G=(X, Y, E)$ be a bipartite graph.Let $S \subseteq X$. Let $u \in S$. A vertex $v$ is a private $X$-neighbor of $u$ with respect to $S$ if $u$ is the only point of $S$, $X$-adjacent to $v$.

A set $S$ is $X$-irredundant set if every $u \in S$ has a private X-neighbor. The $X$-irredundance number of a graph $G$ is the cardinality of a maximal $X$-irredundant set of vertices with minimum cardinality and is denoted by $\operatorname{ir}_{X}(G)$. The upper $X$-irredundance number of a graph $G$ is the cardinality of a $X$-irredundant set of vertices with maximum cardinality and is denoted by $I R_{X}(G)$.

Theorem:3.4.1 A $X$-dominating set $S$ is a minimal $X$-dominating set if and only if it is $X$-dominating and $X$-irredundant.

Proof: Let $S$ be a $X$-dominating set. Then $S$ is a minimal $X$-dominating set if and only if for every $u \in S$ there exists $v \in X-(S-\{u\})$ which is not $\mathrm{X}$-dominated by $S-\{u\}$. Equivalently, $S$ is a minimal $X$-dominating set if and only if $\forall u \in S, u$ has atleast one private X-neighbor with respect to $S$. Thus $S$ is minimal $X$-dominating set if and only if it is $X$-irredundant.

Conversely, Let $S$ is both $X$-dominating and $X$-irredundant.

Claim: $S$ is a minimal $X$-dominating set.

If $S$ is not a minimal $X$-dominating set, then there exists $v \in S$ for which $S-\{v\}$ is $X$-dominating. Since $S$ is $X$-irredundant, $v$ has a private $\mathrm{X}$-neighbor of with respect to $S$ say $u$ ( $u$ may be equal to $v$ ). By definition, $u$ is not X-adjacent to any vertex in $S-\{v\}$. Therefore, $S-\{v\}$ is not a $X$-dominating set, a contradiction. Hence, $S$ is a minimal $X$-dominating 
set.

Theorem:3.4.2 Every minimal $X$-dominating set is a maximal $X$-irredundant set.

Proof: Every minimal $X$-dominating set $S$ is $X$-irredundant set.

Claim: $S$ is a maximal $X$-irredundant set.

Suppose $S$ is not a maximal $X$-irredundant set. Then there exists a vertex $u \in X-S$ for which $S \cup\{u\}$ is $X$-irredundant. Therefore, there exists atleast one vertex $x$ which is a private $X$-neighbor of $u$ with respect to $S \cup\{u\}$. Hence, no vertex in $S$ is $X$-adjacent to $x$. Thus $S$ is not $X$ dominating set, a contradiction. Hence, $S$ is maximal $X$-irredundant set.

A vertex $v$ is a private neighbor of a vertex $u$ in a set $S \subseteq V(G)$ with respect to $S$ if $N[v] \cap S=\{u\}$. The private neighbor set of $u \in S$ with respect to $S$ is defined as $p n[u, S]=\{v: N[v] \cap S=\{u\}\}$. A set $S$ is called irredundant set [2] if for every vertex $u \in S, p n[u, S] \neq \phi$. The irredundance number of agraph $G$ is the cardinality of a maximal irredundant set with minimum number of vertices and is denoted by $\operatorname{ir}(G)$. The upper irredundance number of a graph $G$ is the cardinality of a maximum irredundant set of vertices and is denoted by $I R(G)$.

Theorem:3.4.3 For any graph $G$,

(a) $\operatorname{ir}_{X}(V E(G))=\operatorname{ir}(G)$

(b) $i r_{X}(E V(G))=i r^{1}(G)$

Proof: Let $S$ be a $i r_{X}$ set of $V E(G)=\left(X, Y, E^{1}\right)$. Every $v$ has a private $X$-neighbor $u$. Equivalently, $v$ is $X$-adjacent to $u$ and no other vertex in $S$ is $X$-adjacent to $u$. In $G, v \in S$ is the only vertex adjacent to $u$ and no other vertex in $S$ is adjacent to $u$. Therefore, $S$ is an irredundant set of $G$.

$\operatorname{ir}(G) \leq|S|=i r_{X}(V E(G))$.

Let $U$ be an $i r-$ set of $G$. For every vertex $v \in U, p n[v, U] \neq \phi$. Every vertex $v \in U$ has at least one private neighbor with respect to $u$. In $V E(G)$, that is every vertex $v \in U$ has at least one private $X$-neighbor. Therefore, $U$ is an $X$-irredundant set. Hence, $i r_{X}(\operatorname{VE}(G)) \leq|U|=\operatorname{ir}(G)$. Hence, $i r_{X}(V E(G))=i r(G)$. 
(b) Let $S$ be an $i r_{X}$ set of $E V(G)=\left(X, Y, E^{1}\right)$. Every $e$ has a private $X$-neighbor $f$. Equivalently, $e$ is $X$-adjacent to $f$ and no other vertex in $S$ is $X$-adjacent to $f$. In $G, e \in S$ is the only edge adjacent to $f$ and no other edge in $S$ is adjacent to $f$. Therefore, $S$ is an edge irredundant set of $G$. Hence, $i r^{1}(G) \leq|S|=i r_{X}(E V(G))$.

Let $U$ be a $i r^{1}-$ set of $G$. For every edge $e \in U, p n[e, U] \neq \phi$. Hence, every edge $e \in U$ has at least one private neighbor. That is, in $E V(G)$, every vertex $e \in U$ has at least one private $X$-neighbor. Therefore, $U$ is an $X$-irredundant set in $E V(G)$. Thus, $\operatorname{ir}_{X}(E V(G)) \leq|U|=i r^{1}(G)$. Hence, $i r_{X}(E V(G))=i r^{1}(G)$.

\section{Main Result}

For any graph $G, I R_{v e}(G)+\gamma(G) \leq|V|$ and $\gamma_{v e}(G)+I R(G) \leq|V|$ are proved using bipartite theory of graphs, which are open problem in [3].

Theorem:4.1 Let $G=(X, Y, E)$ be a bipartite graph with $N_{Y}(x) \neq \phi$ for every $x \in X$. Then $I R_{h Y}(G)+\gamma_{X}(G) \leq|X|$.

Proof: Let $S$ be a $I R_{h Y}$ set of $G$. Then, $S$ is a maximal hyper $Y$ irredundant set. Therefore, $S$ is a hyper $Y$-irredundant set. That is every $x \in S$ has a private hyper Y-neighbor $y \in Y$. Then $x$ is adjacent to $y$ or $y \in N\left(N_{Y}(x)\right)$ and for all vertices $x_{1} \in S-\{x\}, x_{1}$ is not adjacent to $y$ and $y \notin N\left(N_{Y}(x)\right)$.

Case(i): $x$ is adjacent with $y$.

Since $N_{Y}(v) \neq \phi, x$ has $X$-neighbours. Let $z$ be any $X$-neighbour of $x$. Suppose $z \in S$. Then $z$ is not adjacent to $y$ and $y \notin N\left(N_{Y}(z)\right)$. But $y \in N\left(N_{Y}(x)\right)$, since $x$ is a $X$-neighbour of $z$, a contradiction. Therefore, any $X$-neighbour of $x$ is in $X-S$.

Case(ii): $y \in N\left(N_{Y}(x)\right)$.

Vertices in $N(y)$ are in $X-S$. Then $N(y) \subseteq X-S$. Other wise, we get a contradiction to $y \in Y$ is a private hyper $Y$-neighbor of $x \in S$. Hence, for every $x \in S$ there exists $x_{1} \in X-S$ such that $x$ and $x_{1}$ are $X$-adjacent. That is, $X-S$ is a $X$-dominating set. Therefore, $\gamma_{X}(G) \leq|X-S|=$ $|X|-I R_{h Y}(G)$. Hence, $I R_{h Y}(G)+\gamma_{X}(G) \leq|X|$. 
Corollary: 4.2 For any graph $G$,

(a) $I R_{v e}(G)+\gamma(G) \leq|V|$

(b) $I R_{e v}(G)+\gamma_{1}(G) \leq|E|$.

Theorem:4.3 Let $G=(X, Y, E)$ be a bipartite graph with $N_{Y}(x) \neq \phi$ for every $x \in X$ then $I R_{X}(G)+\gamma_{h Y}(G) \leq|X|$.

Proof: Let $S$ be a $I R_{X}$ set of $G$. Every element $x \in S$ has a private $X$-neighbor. Consider the set $X-S$. Since $X-S$ is a $X$-dominating set elements of $Y$ are either adjacent to $X-S$ or adjacent to vertices which are $X$-adjacent to elements of $X-S$. Therefore, $X-S$ is a hyper $Y$-dominating set. Therefore, $\gamma_{h Y} \leq|X-S|=|X|-I R_{X}$. Hence, $I R_{X}+\gamma_{h Y} \leq|X|$.

Corollary: 4.4 For any graph $G$,

(a) $\gamma_{v e}(G)+I R(G) \leq|V|$

(b) $\gamma_{e v}(G)+I R^{1}(G) \leq|E|$.

Acknowledgement: We are thankful to the anonymous referee for helpful suggestions, which led to substantial improvement in the paper.

\section{References}

[1] Bondy J. A., Murthy U. S. R., Graph theory with applications, London Macmillan (1976).

[2] Haynes T. W., Hedetniemi. S. T. and Slater P. J., Fundamentals of Domination in graphs, Marcel Dekker, New York, (1998).

[3] Jason Robert Lewis, Vertex-edge and edge-vertex parameters in graphs, (Ph. D Thesis), Clemson University, August 2007.

[4] Stephen Hedetniemi, Renu Laskar, A Bipartite theory of graphs I, Congressus Numerantium, Volume 55; pp. 5-14, December 1986.

[5] Stephen Hedetniemi, Renu Laskar, A Bipartite theory of graphs II, Congressus Numerantium, Volume 64; pp. 137-146, November 1988. 
[6] Swaminathan V. and Venkatakrishnan Y. B., Hyper $Y$-domination in Bipartite graphs, International Mathematical Forum, Volume 4, No. 20, pp. 953-958, (2009).

\section{Swaminathan ${ }^{a}$}

Research Coordinator,

Ramanujan Research Centre,

S. N. College,

Madurai,

India

e-mail : sulanesri@yahoo.com

and

Y. B. Venkatakrishnan ${ }^{b}$

Department of Mathematics,

SASTRA University,

Tanjore,

India

e-mail : venkatakrish2@maths.sastra.edu 